\title{
BUILDING VERY LARGE NEIGHBOUR-JOINING TREES
}

\author{
Martin Simonsen, Thomas Mailund, Christian N. S. Pedersen \\ Bioinformatics Research Center (BIRC), Aarhus University, C. F. Mфllers Allé 8, DK-8000 Århus C, Denmark. \\ zxr@birc.au.dk,mailund@birc.au.dk,cstorm@birc.au.dk
}

Keywords: Neighbour-joining, distance based phylogenetic inference, I/O algorithms, RapidNJ, evolution.

\begin{abstract}
The neighbour-joining method by Saitou and Nei is a widely used method for phylogenetic reconstruction, made popular by a combination of computational efficiency and reasonable accuracy. With its cubic running time by Studier and Kepler, the method scales to hundreds of species, and while it is usually possible to infer phylogenies with thousands of species, tens or hundreds of thousands of species is infeasible. Recently we developed a simple branch and bound heuristic, RapidNJ, which significantly reduces the average running time. However, the $O\left(n^{2}\right)$ space consumption of the RapidNJ method, and the NJ method in general, becomes a problem when inferring phylogenies with $10000+$ taxa.

In this paper we present two extentions of RapidNJ which reduce memory requirements and enable RapidNJ to infer very large phylogenetic trees efficiently. We also present an improved search heuristic for RapidNJ which improves RapidNJ's performance on many data sets of all sizes.
\end{abstract}

\section{INTRODUCTION}

The neighbour-joining (NJ) method (Saitou and Nei, 1987) is a widely used method for phylogenetic inference, made popular by reasonable accuracy combined with a cubic running time by Studier and Kepler (Studier and Kepler, 1988). The NJ method scales to hundreds of species, and while it is usually possible to infer phylogenies with thousands of species, tens or hundreds of thousands of species is computationally infeasible.

Implementations like QuickTree (Howe et al., 2002) and QuickJoin (Mailund et al., 2006; Mailund and Pedersen, 2004) use various approaches to reduce the running time of $\mathrm{NJ}$ considerably, and recently we presented a new heuristic, RapidNJ (Simonsen et al., 2008), which uses a simple branch and bound technique to reduce the running time even further. Though RapidNJ is able to build $\mathrm{NJ}$ trees very efficiently it requires, like the canonical $\mathrm{NJ}$ method, $\mathrm{O}\left(n^{2}\right)$ space to build a tree with $n$ taxa. The space consumption of RapidNJ, and the NJ method in general, is thus a practical problem when building large trees, and since RapidNJ uses some additional data structures of size $\mathrm{O}\left(n^{2}\right)$, this method has limited application to data sets with more than 10,000 taxa which is of interest when building phylogenetic trees from e.g. Pfam (Finn et al., 2006) sequence data.

In this paper we present two extensions for RapidNJ which reduce the memory requirements of RapidNJ. The first extension uses a simple heuristic which takes advantage of RapidNJ's memory access pattern to reduce the internal memory (RAM) consumption. The second extension is based on the first extension and makes use of external memory, i.e. a hard disk drive (HDD) to alleviate internal memory consumption. We also present an improved version of the search heuristic used in RapidNJ which increases performance on data sets that RapidNJ has difficulties handling.

The two extensions combined with the improved search heuristic allow RapidNJ to build large NJ trees efficiently which is important as sequence family data with more than 50,000 taxa are becoming widely available (Finn et al., 2006; Alm et al., 2005). Also, the NJ method is used as a clustering method in both micro array data analysis and metagenomics where data sets can become very large. Using the methods 
proposed in this paper, clustering of large data sets can be handled efficiently on normal desktop computers.

We evaluate the performance of the extended RapidNJ method (ERapidNJ), by comparing running times of an implementation of the ERapidNJ method with other fast implementations for building canonical NJ trees.

\section{METHODS}

\subsection{The Neighbour-Joining Method}

$\mathrm{NJ}$ is a hierarchical clustering algorithm. It takes a distance matrix $D$ as input, where $D(i, j)$ is the distance between clusters $i$ and $j$. Clusters are then iteratively joined using a greedy algorithm, which minimises the total sum of branch lengths in the tree. Basically the algorithm uses $n$ iterations, where two clusters $(i, j)$ are selected and joined into a new cluster in each iteration. The pair $(i, j)$ is selected by minimising

$$
Q(i, j)=D(i, j)-u(i)-u(j),
$$

where

$$
u(l)=\sum_{k=0}^{r-1} D(l, k) /(r-2),
$$

and $r$ is the number of remaining clusters. When a minimum q-value $q_{\min }=\min _{0 \leq i, j<r} Q(i, j)$ is found, $D$ is updated, by removing the $i$ 'th and $j$ 'th row and column. A new row and a new column are inserted with the distances to the new cluster. The distance between the new cluster $a=i \cup j$ and one of the remaining clusters $k$, is calculated as

$$
D(a, k)=\frac{D(i, k)+D(j, k)-D(i, j)}{2} .
$$

The result of the algorithm is an unrooted bifurcating tree where the initial clusters correspond to leafs and each join corresponds to inserting an internal node in the tree.

\subsection{RapidNJ}

RapidNJ (Simonsen et al., 2008) computes an upper bound for the distance between clusters which is used to exclude a large portion of $D$ when searching for a minimum q-value. To utilise the upper bound two new data structures, $S$ and $I$, are needed. Matrix $S$ contains the distances from $D$ but with each row sorted in increasing order and matrix $I$ maps the ordering in
$S$ back to positions in $D$. Let $o_{1}, o_{2}, \ldots, o_{n}$ be a permutation of $1,2, \ldots, n$ such that $D\left(i, o_{1}\right) \leq D\left(i, o_{2}\right) \leq$ $\cdots \leq D\left(i, o_{n}\right)$, then

$$
S(i, j)=D\left(i, o_{j}\right)
$$

and

$$
I\left(i, o_{j}\right)=j .
$$

The upper bound is computed and used to speed up the search for a minimum q-value as follows.

1. Set $q_{\min }=\infty, i=-1, j=-1, u_{\max }=\max (u(l))$

2. for each row $r$ in $S$ and column $c$ in $r$ :

(a) if $S(r, c)-u(r)-u_{\max }>q_{\min }$ then move to the next row.

(b) if $Q(r, I(r, c))<q_{\text {min }}$ then set $q_{\min }=$ $Q(r, I(r, c)), i=r$ and $j=I(r, c)$.

The algorithm searches $S$ row-wise and stops searching within a row when the condition

$$
S(r, c)-u(r)-u_{\max }>q_{\min }
$$

is true, or the end of a row is reached. If we reached an entry in $S$ where (6) is true, we are looking at a pair $(i, j)$, where $D(i, j)$ is too large for $(i, j)$ to be a candidate for $q_{\mathrm{min}}$, and because $S$ is sorted in increasing order, all the following entries in $S(i)$ can now be disregarded in the search.

When the cluster-pair $\left(i^{\prime}, j^{\prime}\right)$ with the minimum qvalue is found, $D$ is updated as described in Sec. 2.1. The $S$ and $I$ matrices are then updated to reflect the changes made in the $D$ as follows. Row and column $i^{\prime}$ and $j^{\prime}$ are marked as deleted and entries in $S$ belonging to these rows/columns are then identified using $I$ and ignored in the following iterations of the NJ method. A new row containing the distances of the new cluster is sorted and inserted into $S$.

\subsection{Reducing the Memory Consumption of RapidNJ}

RapidNJ consumes about four times more memory than a straightforward implementation of canonical neighbour-joining, which makes it impractical to use on large data sets. We propose an extension to RapidNJ which reduces the memory consumption significantly while only causing a small reduction in performance.

First we reduce the size of the $D$ matrix. RapidNJ stores the complete $D$ matrix in memory, even though only the upper or lower triangular matrix is needed, because it allows for a more efficient memory access scheme. By only storing the lower triangular matrix, the size of $D$ is halved without affecting performance seriously. 


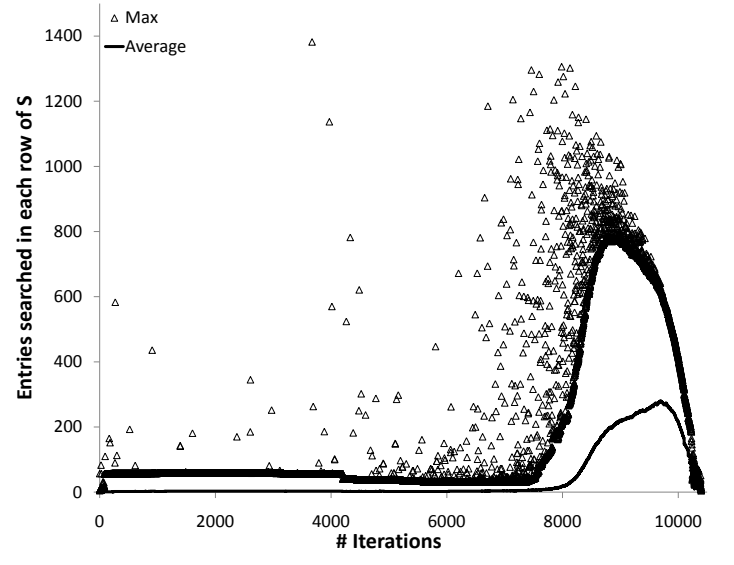

Figure 1: The maximum and average number of entries of each row in $S$ that RapidNJ searched during each iteration of the NJ method when building a typical tree containing 10,403 taxa.

Secondly, the size of $S$ and, consequently, $I$ are reduced. As seen in Fig. 1, RapidNJ rarely needs to search more than a few percent of each row in $S$. Hence it is not necessary to store the full $S$ matrix in memory to receive a speed up similar to the original RapidNJ method. An increase in both maximum and average search depth is observed when the last quarter of the clusters remains, but as the number of remaining clusters is low at this point, the increase only causes a relatively small increase in the total number of entries searched. The size of $S$ is reduced by only storing as many columns of $S$ as can fit in the available internal memory after $D$ has been loaded. Of course we might not store enough columns of $S$ to complete the search for $q_{\min }$ in all rows of $S$, i.e. we might not reach an entry where (6) becomes true. If this happens we simply search the corresponding row in $D$.

There is a lower limit on the number of columns of $S$ we must store before the performance is severely affected, but there is no exact number as it depends on the data set. Our experiments imply that at least 5\% of the columns in $S$ are needed to receive a significant speed up in general.

\subsection{An I/O Algorithm for Building Very Large Trees}

Even when using the extension described in Sec. 2.3 RapidNJ will run out of memory at some point and begin to swap out memory pages to the HDD. This will seriously reduce the performance because the data structures used by RapidNJ are not designed to be I/O efficient. I/O efficiency is achieved by accessing data in the external memory in blocks of typical 4-8 KB corresponding to the block size $B$ of the HDD used (Aggerwal and Vitter, 1988), and it is often bet- ter to access data in blocks larger than $B$ to take full advantage of hardware and software caching. However, even when using an I/O efficient algorithm, accessing data in the external memory has very high latency compared to accessing data in the internal memory, thus external memory data access should be kept at a minimum.

RapidDiskNJ is an extension to RapidNJ which employs both internal and external memory storage efficiently. Because RapidNJ only uses $S$ (and $I$ ) to search for $q_{\mathrm{min}}, D$ can be stored in the external memory without affecting performance significantly. Furthermore, as explained in Sec. 2.3, RapidNJ usually only needs to search a small fraction of $S$ in each iteration, so the total internal memory consumption can be reduced by only representing a sufficient part of $S$ in the internal memory. Using external memory to store $D$ affects the running time by a large but constant factor, thus RapidDiskNJ has the same $O\left(n^{3}\right)$ asymptotic running time as RapidNJ. $q_{\min }$ is found as described in Sec. 2.3 the only difference being that searching $D$ is done using the external memory.

\subsubsection{Data Structures}

$D$ is stored row-wise in the external memory, so all access to $D$ must be done row-wise as accessing a column of $D$ would result in $r$ I/O operations (read/write operations) assuming that an entry in $D$ has size $\leq B$. A row in $D$ can be accessed using $\frac{r * \alpha}{B} \mathrm{I} / \mathrm{O}$ operations where $\alpha$ is the size of an entry in $D$, which is much more efficient.

As explained in Sec. 2.3 storing half of $D$ is sufficient, but by storing the whole $D$-matrix in the external memory, all distances from one cluster to all other clusters can be accessed by reading one row of $D$. After each iteration of the NJ method, at least one column of $D$ needs to be updated with new distances after a join of two clusters. This would trigger column-wise external memory access but by using an internal memory cache this can be avoided as described below. Deletion of columns in $D$ is done in $O(1)$ time by simply marking columns as deleted and then ignoring entries in $D$ belonging to deleted columns. This gives rise to a lot of "garbage" in $D$, i.e. deleted columns, which needs to be removed to avoid a significant overhead. In Sec. 2.4.2 an efficient garbage collection strategy to handle this problem is proposed.

RapidDiskNJ builds the $S$-matrix by sorting $D$ row by row and for each sorted row the first $\frac{1}{\gamma}$ entries are stored in the internal memory where the size of $\frac{n}{\gamma}$ is $\frac{M}{2}$ and $M$ is the size of the internal memory. If enough columns of $S$ can be stored in the internal memory, 
RapidDiskNJ can usually find $q_{\text {min }}$ using only $S$ which means that RapidDiskNJ rarely needs to access the external memory.

The other half of the internal memory is used for caching columns of $D$. After each iteration a new column for $D$ is created but instead of inserting this in $D$, The column is stored in an internal memory cache $C$. By keeping track of which columns have been updated and in which order, updated entries in $D$ can quickly be identified and read from $C$. When $C$ is full (i.e. the size has reached $\frac{M}{2}$ ), all updated values in $C$ are flushed to $D$, by updating $D$ row by row which is more efficient than writing columns to $D$ when $C$ is large.

\subsubsection{Garbage Collection}

Entries belonging to deleted columns are left in both $D$ and $S$ after clusters are joined. We just skip these entries when we meet them. This is not a problem for small data sets but in larger data sets they need to be removed to keep $S$ and $D$ as small as possible. Garbage collection in both $D$ and $S$ is expensive so RapidDiskNJ only performs garbage collection when $C$ is flushed. During a flush of $C$, all rows in $D$ are loaded into the internal memory where deleted entries can be removed at an insignificant extra cost. By removing entries belonging to both deleted rows and columns the size of $D$ is reduced to $r$ which makes both searching $D$ and future flushes of $C$ more efficient.

Garbage collection in $S$ is performed by completely rebuilding $S$ during a flush of $C$. Our experiments showed that rebuilding $S$ each time we flush $C$ actually decreases performance because of the time it takes to sort $D$. We found that the best average performance was achieved if $S$ was rebuild only when more than half of $S$ consisted of garbage. During garbage collection of $S$ the number of rows in $S$ decreases to $r$, which allows more columns to be added to $S$ so that $S$ attains size $\frac{M}{2}$ again.

\subsection{Improving the Search Heuristic}

RapidNJ uses the maximum average row sum $u_{\max }$ to compute an upper bound on q-values. Initially row $i$ in $S$ only needs to contain $i$ columns so a tighter bound can be computed if $u_{\max }$ is computed for each row in $S$ i.e. $u(i)_{\max }=\max _{0 \leq l \leq i}(u(l))$. For each new row $i^{\prime}$ created after a join we assign $u\left(i^{\prime}\right)_{\max }=$ $\max _{0 \leq l \leq r}(u(l))$. Updating the existing $u(i)_{\max }$ values can be done by updating $u$-values in the same order as the rows of $S$ were created, assuming that the initial rows of $S$ were created in the order, shortest to longest. Now $u(i)_{\max }=u_{\max }^{\prime}$ where $u_{\max }^{\prime}$ is the largest

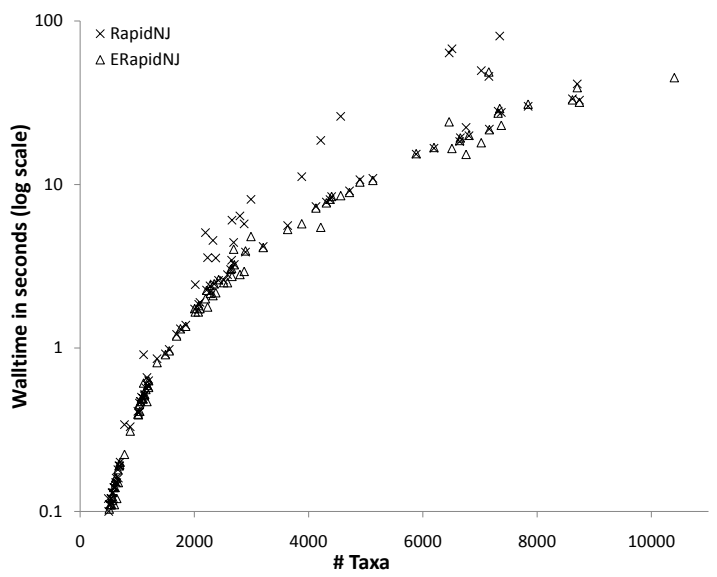

Figure 2: The difference in running time between the original RapidNJ search heuristic and the improved search heuristic. We observe that many of the outliers have been removed when using the improved bounds on q-values and efficient redundant data handling.

$\mathrm{u}$-value seen when $u(i)$ is updated. This takes time $O(r)$. The tighter bounds are very effective on data sets containing cluttering of taxa (where a group of taxa has almost identical distances to each other and a small or zero mutual distance), which gave rise to poor performance in RapidNJ (see Fig. 2).

Redundant data (taxa with equal distances to all other taxa and a mutual distance of 0) is quite common in Pfam data sets. Redundant data often causes a significant loss of performance in RapidNJ because a lot of $q$-values fall under the upper bound at the same time forcing RapidNJ to search all pairs of redundant taxa in each iteration until they are joined. To address this problem we initially treat redundant taxa as a single taxon. When a cluster representing such a taxon is selected for a join, we only delete the cluster if the number of redundant taxa it represents drops to 0 . Identifying and processing redundant taxa can be done in $O\left(n^{2}\right)$ time in a preprocessing phase and reduces the problem of redundant taxa considerably (see Fig. 2).

\section{EXPERIMENTS}

The methods described in Sec. 2 were used to extend the original RapidNJ tool and create the ERapidNJ tool (Source code available at http:// birc.au.dk/Software/RapidNJ/). To assess the performance of the ERapidNJ tool, we compared running times on Pfam data sets with running times of three other tools which to our knowlegde are the fastest tools available for computing canonical NJ 
trees at the moment.

- QuickTree (Howe et al., 2002): An efficient implementation of the NJ method with a heuristic for handling redundant data.

- QuickJoin (Mailund and Pedersen, 2004): Reduces the running time of the NJ method by using information from previous iterations of the $\mathrm{NJ}$ method to reduce the search space significantly.

- NINJA (Wheeler, 2009): Uses an upper bound on q-values like RapidNJ but computes a tighter bound. NINJA also uses the same technique as QuickJoin to further reduce the search space and can also utilise external memory efficiently.

QuickTree is implemented in $C$, QuickJoin and ERapidNJ in $C++$ while NINJA is implemented in Java.

Tools such as Fast Neighbor-Joining (Elias and Lagergren, 2005), Clearcut (Sheneman et al., 2006) and FastTree (Price et al., 2009) which modify the NJ optimisation criteria are not included in the experiments. FastTree is able to construct large trees efficiently, but as this tool operates on multiple alignments and not on distance matrices a direct comparison of the performance of ERapidNJ and FastTree is difficult. See (Simonsen et al., 2008) and (Price et al., 2009) for a comparison of the original RapidNJ tool and some of these tools.

The data used in our experiments is distance matrices computed by QuickTree using multiple alignments from the Pfam database.

The ERapidNJ tool automatically chooses one of three methods for building trees, based on the memory requirements of a given data set and the available amount of memory in the system. For small data sets the original RapidNJ method is used, for medium sized data sets the method described in Sec. 2.3 is used to reduce the memory consumption and for large inputs RapidDiskNJ described in Sec. 2.4 is used. The improved search heuristic described in Sec. 2.5 is used in all three methods to limit the impact of redundant data and reduce the search space.

NINJA, like the ERapidNJ tool, is capable of using both internal and external memory. In the experiments NINJA was configured to use only internal memory for data sets which could fit in the 2 GB memory. For larger data sets NINJA used both internal and external memory.

\subsection{Experimental Setup}

All experiments were performed on machines with an Intel Core $266002.4 \mathrm{GHz}$ CPU, 2 GB $667 \mathrm{MHz}$ RAM and a 7200 RPM 160 GB, Western Digital

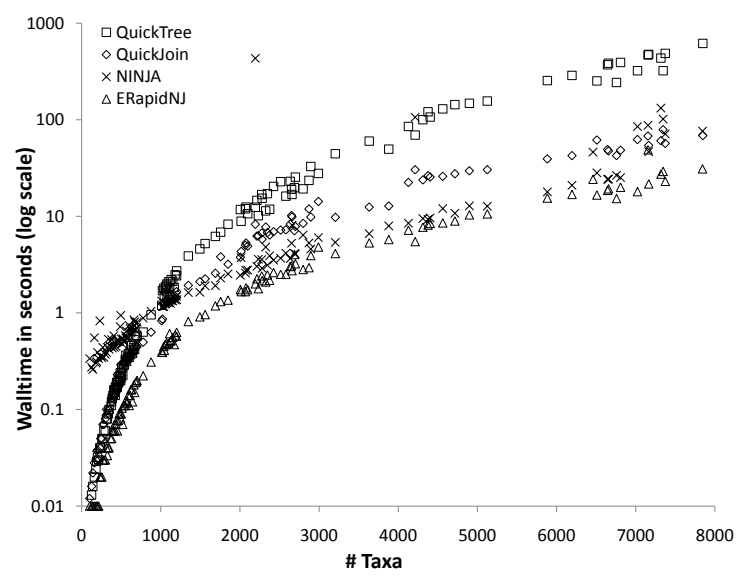

Figure 3: Running times on data sets with 100 to 8,000 taxa.

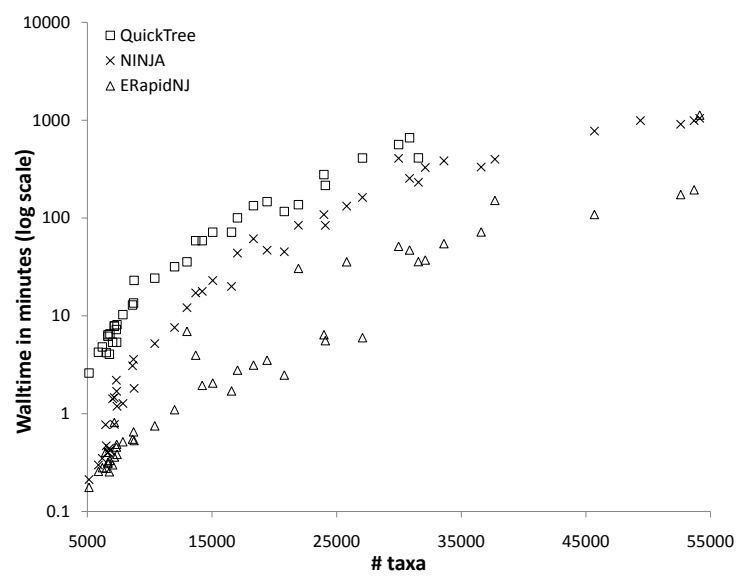

Figure 4: Running times on data sets with 5,000 to 55,000 taxa.

HDD. The operating system was Red Hat Enterprise 5.232 bit with Java 1.6 installed.

\subsection{Results and Discussion}

As seen in Fig. 3, ERapidNJ is faster than the three other tools on data sets up to 3,000 taxa. NINJA seems to suffer from an overhead on small data sets which is probably caused by the time required to initialise Java and the data structures NINJA needs, which are larger and more complex than those used by ERapidNJ. Except for a few outliers from NINJA, ERapidNJ and NINJA have roughly the same running time on data sets with 3,000 to 7,000 taxa. On data sets with more than 7,000 taxa NINJA runs out of internal memory and starts using external memory. Both QuickJoin and QuickTree are consistently slower than ERapidNJ and QuickJoin runs out of memory on data sets with more than 7,000 taxa like NINJA. 
Figure 4 shows running times on data sets with 5,000 to 55,000 taxa. Because ERapidNJ is able to scale its memory consumption to the size of the data set, we observe that ERapidNJ is significantly faster than NINJA on data sets containing less than 28,000 taxa. On larger data sets ERapidNJ is still much faster than NINJA on most data sets, and we only found two large data sets where NINJA outperformed ERapidNJ. One of these results (a data set with 49,376 taxa) is not shown in Fig. 4 for ERapidNJ because ERapidNJ did not finish within 48 hours due to cluttering of data. NINJA was able to finish this data set in 16 hours because NINJA computes much tighter bounds on q-values than ERapidNJ. NINJA also uses a technique called q-filtering (Wheeler, 2009) to further reduce the search space when searching for $q_{\min }$. This is computationally expensive but on a few large data sets with cluttering the tighter bounds give NINJA an advantage because ERapidNJ cannot store enough columns of $S$ in the internal memory. More memory improves ERapidNJs performance on these data sets significantly.

The performance of QuickTree was inferior to both NINJA and ERapidNJ on all data sets. When trying to build trees with more than 32,000 taxa using QuickTree the running time exceeded 48 hours because more than 2GB of internal memory is needed to build such trees which results in memory page swapping. Since QuickTree is not I/O efficient, page swapping causes a huge penalty which prevents QuickTree from finishing within a resonable amount of time.

\subsubsection{Improving Performance by Parallelisation}

Parallisation of the original NJ method can be done by dividing the rows of $D$ into $t$ sets of approximately the same size and then searching each set for $q_{\text {min }}$ in parallel. Similarly, ERapidNJ can be parallised by searching rows of $S$ in parallel. The performance of the canonical NJ method can easily be improved in this way, as searching for $q_{\min }$ is the most time consuming step of the canonical NJ method. This is not always the case with ERapidNJ where the time spent on operations such as reading the distance matrix from the HDD, sorting $S$ and updating data structures is similar to the total time used on searching for $q_{\text {min }}$ when building relatively small trees. As an example, ERapidNJ uses $33 \%$ of the total running time on reading the distance matrix and only $24 \%$ of the total running time on searching for $q_{\min }$ when building a tree containing 10,403 taxa. Operations such as reading data from external memory and updating data structures in internal memory does not benefit significantly from parallelisation and consequently limits the potential performance gain from parallelisation of
ERapidNJ in the case of small data sets. On larger data sets the total time used to search for $q_{\min }$ takes up a substantial part of the total time consumption and here parallelisation is more effective.

Experiments with a parallelised version of ERapidNJ showed a reduction of the total running time by a factor 2.2 on a quad core Intel Core 2 Duo processor compared to the unparallised ERapidNJ on the same processor when building a tree with 25,803 taxa. When building a tree with 10,403 taxa the total running time was only reduced by a factor 1.12 . Both these data sets were computed in internal memory and parallelisation of RapidDiskNJ will not increase performance significantly as RapidDiskNJ is I/O bound, i.e. most of the running time is spend on waiting for the external memory.

\section{CONCLUSIONS}

We have presented two extensions and an improved search heuristic for the RapidNJ method which both increases the performance of RapidNJ and decreases internal memory requirements significantly. Using the methods described in this paper, we were able to overcome RapidNJs limitations regarding the memory consumption and performance on data sets containing redundant and cluttered taxa. We have presented experiments with the extended RapidNJ tool showing that canonical $\mathrm{NJ}$ trees containing more than 50,000 taxa can be build in a few hours on a desktop computer with only $2 \mathrm{~GB}$ of RAM. Our experiments also showed that in comparison with the fastest tools available, for building canonical $\mathrm{NJ}$ trees, the ERapidNJ tool is significantly faster on any size of input.

We are aware that statistical phylogenetic inference methods with better precision than distance based method are available. However, the time complexity of these methods are high compared to the NJ method and currently they do not scale well to large data sets (Stamatakis, 2006; Ott et al., 2007), which justify the existence of distance based methods as presented in this paper.

\section{REFERENCES}

Aggerwal, A. and Vitter, T. S. (1988). The input output complexity of sorting and related problems. In Communications of the ACM, volume 31(9), pages 11161127.

Alm, E. J., Huang, K. H., Price, M. N., Koche, R. P., Keller, K., Dubchak, I. L., and Arkin, A. P. (2005). The 
microbesonline web site for comparative genomics. Genome Research, 15(7):1015-1022.

Elias, I. and Lagergren, J. (2005). Fast neighbour joining. In Proceedings of the 32nd International Colloquium on Automata, Languages and Programming (ICALP), volume 3580 of Lecture Notes in Computer Science, pages 1263-1274. Springer.

Finn, R. D., Mistry, J., Schuster-Böckler, B., GriffithsJones, S., Hollich, V., Lassmann, T., Moxon, S., Marshall, M., Khanna, A., Durbin, R., Eddy, S. R., Sonnhammer, E. L. L., and Bateman, A. (2006). Pfam: clans, web tools and services. Nucleic Acids Research, Database Issue 34:D247-D251.

Howe, K., Bateman, A., and Durbin, R. (2002). QuickTree: Building huge neighbour-joining trees of protein sequences. Bioinformatics, 18(11):1546-1547.

Mailund, T., Brodal, G. S., Fagerberg, R., Pedersen, C. N. S., and Philips, D. (2006). Recrafting the neighborjoining method. BMC Bioinformatics, 7(29).

Mailund, T. and Pedersen, C. N. S. (2004). QuickJoin - fast neighbour-joining tree reconstruction. Bioinformatics, 20:3261-3262.

Ott, M., Zola, J., Stamatakis, A., and Aluru, S. (2007). Large-scale maximum likelihood-based phylogenetic analysis on the ibm bluegene/l. In Proceedings of the 2007 ACM/IEEE conference on Supercomputing, pages 1-11.

Price, M. N., Dehal, P. S., and Arkin, A. P. (2009). Fasttree: Computing large minimum-evolution trees with profiles instead of a distance matrix. Mol Biol Evol, 26(7):1641-1650.

Saitou, N. and Nei, M. (1987). The neighbor-joining method: A new method for reconstructing phylogenetic trees. Molecular Biology and Evolution, 4:406425.

Sheneman, L., Evans, J., and Foster, J. A. (2006). Clearcut: A fast implementation of relaxed neighbor-joining. Bioinformatics, 22(22):2823-2824.

Simonsen, M., Mailund, T., and Pedersen, C. N. S. (2008). Rapid neighbour-joining. In Algorithms in Bioinformatics, Proceedings 8th International Workshop, WABI 2008, volume 5251, pages 113-123.

Stamatakis, A. (2006). Raxml-vi-hpc: maximum likelihood-based phylogenetic analyses with thousands of taxa and mixed models. Oxford Journals, 22(21):2688-2690.

Studier, J. A. and Kepler, K. J. (1988). A note on the neighbour-joining method of Saitou and Nei. Molecular Biology and Evolution, 5:729-731.

Wheeler, T. J. (2009). Large-scale neighbor-joining with ninja. In Algorithms in Bioinformatics, Proceedings 9th International Workshop, WABI 2009, volume 5724/2009, pages 375-389. 